Original Article

\title{
The Effects of Closed Kinetic Chain Exercises and Open Kinetic Chain Exercises Using Elastic Bands on Electromyographic Activity in Degenerative Gonarthritis
}

\author{
Igsoo Cho, MS, PT ${ }^{1)}$, Gak Hwangbo, PhD, PT ${ }^{2)}$, Daenee Lee, PhD, $\mathrm{PT}^{3)}$, \\ SANGYONG LeE, PhD, $\mathrm{PT}^{3)^{*}}$ \\ 1) Department of Rehabilitation Science, Graduate School of Daegu University, Republic of Korea \\ 2) Department of Physical Therapy, College of Rehabilitation Science, Daegu University, Republic of \\ Korea \\ 3) Department of Physical Therapy, Youngdong University: 12 Youngdong-eup, Youngdong-gun, \\ Chungbuk 370-701, Republic of Korea
}

\begin{abstract}
Purpose] The purpose of this study was to examine the effects of closed kinetic chain exercises (CK$\mathrm{CEs}$ ) and open kinetic chain exercises (OKCEs) with elastic bands on the electromyographic activity of patients with degenerative gonarthritis. [Subjects] The study subjects were 30 degenerative gonarthritis patients who were divided into a CKCE group (CKCEG, $n=10)$, an OKCE group (OKCEG, $n=10)$, and a control group $(C G, n=10)$. [Methods] The CKCEG and the OKCEG performed exercises with elastic bands, and the CG took part in a quadriceps strengthening exercise. All three groups performed the exercises three times per week for four weeks. The electromyographic activities of the subjects' vastus medialis (VM), rectus femoris (RF), vastus lateralis (VL), semitendinosus (ST), and biceps femoris (BF) muscles were measured and compared. [Results] Within-group comparisons revealed that the electromyographic activities of the VM, RF, VL, ST, and BF muscles increased significantly in the CKCEG. The OKCEG displayed significant increases in the electromyographic activity of the VM, RF, ST, and BF muscles, and the CG showed significant increases in the electromyographic activities of the RF, VL, ST, and $\mathrm{BF}$ muscles. In between-group comparisons after the intervention, the electromyographic activities of the VM, RF, and VL muscles of the CKCEG were significantly higher than those of the CG. The electromyographic activities of the VM, RF, and ST muscles of the OKCEG were significantly higher than those of the CG. [Conclusion] We consider CKCEs with elastic bands are an effective intervention for increasing the electromyographic activities of the $\mathrm{VM}, \mathrm{RF}, \mathrm{VL}, \mathrm{ST}$, and BF muscles of degenerative gonarthritis patients, and OKCEs with elastic bands are an effective intervention for increasing the electromyographic activities of the VM, RF, ST, and BF muscles of degenerative gonarthritis patients.

Key words: Elastic-band, Close kinetic chain exercise, Open kinetic chain exercise
\end{abstract}

(This article was submitted Feb. 13, 2014, and was accepted Mar. 31, 2014)

\section{INTRODUCTION}

The prevalence rate of chronic degenerative arthritis is very high. Among elderly persons aged 65 years or more, females are estimated to have two times higher prevalence rates of chronic degenerative arthritis than males, and most elderly persons have degenerative arthritis ${ }^{1}$. The physical characteristics of chronic degenerative gonarthritis include pain, deformation, and edema. These physical characteristics lead to postural deformation. Long-term postural deformation causes the muscles to become fixed and rigid, result-

*Corresponding author. Sangyong Lee (E-mail: 1sy8275@ hanmail.net)

(C) The Society of Physical Therapy Science. Published by IPEC Inc.

This is an open-access article distributed under the terms of the Creative Commons Attribution Non-Commercial No Derivatives (by-ncnd) License $<$ http://creativecommons.org/licenses/by-nc-nd/3.0/>. ing in reduced flexibility and abnormal gait. The effects of gonarthritis lead not only to a lack of activity and exercise, but also to a deterioration in patients' physical fitness, forming a vicious circle ${ }^{2}$. Chronic degenerative arthritis leads to marked weakening of the quadriceps femoris extensor muscle ${ }^{3)}$. The quadriceps femoris muscle is a major kneejoint stabilizing muscle. Together with age, atrophy of this muscle results in knee pain and functional impairment. Performing exercises to strengthen this muscle can relieve pain and lead to functional recovery of the muscle ${ }^{4)}$.

Chronic degenerative arthritis is treated with drug therapies, nonpharmacological therapies, and surgery. Recently, among nonpharmacological therapies, exercise therapies have been frequently used to improve patients' physical functions. Among exercise therapies, resistive exercises using bands have been reported to be very effective for elderly persons ${ }^{5,6)}$. Such exercises stimulate the proprioceptive senses of the joints and muscles to transmit information 
on the location and the movement of the joints to the cerebrum $^{5,6)}$. In this way, correct posture is maintained, and little stress is imposed on the joints, even when exercises are performed using diverse ranges of joint motion ${ }^{5,6}$ )

In general, when elastic bands are used in CKCEs, the aim is to improve muscle strength. They are frequently used in OKCEs due to their convenient use. When elastic bands are used at home or in rehabilitation facilities, OKCEs are favored over CKCEs because they are more convenient to use. CKCEs and OKCEs are customarily used when elastic bands are used without any concrete evidence for difference between them. Although many previous studies of CKCEs and OKCEs using elastic bands have reported the effects of the exercise on degenerative gonarthritis patients, few studies have examined their effects on the electromyographic activity. Therefore, this study investigated the effects of CKCEs and OKCEs using elastic bands on the electromyographic activities of the leg muscles of patients with degenerative gonarthritis.

\section{SUBJECTS AND METHODS}

The study subjects were 30 elderly persons aged 60 years or more who had been diagnosed with degenerative gonarthritis. We selected them from among patients visiting $\mathrm{G}$ hospital located in Gumi, Gyeongsangbuk-do. The mean age of the CKCEG $(n=10)$ was $67.20 \pm 7.36$ years, the mean height was $155.10 \pm 4.48 \mathrm{~cm}$, and the mean weight was $56.90 \pm 9.09 \mathrm{~kg}$. The OKCEG $(\mathrm{n}=10)$ mean age was $65.50 \pm 5.85$ years, the mean height was $156.50 \pm 8.46 \mathrm{~cm}$, and the mean weight was $62.80 \pm 5.97 \mathrm{~kg}$. The CG $(\mathrm{n}=10)$ mean age was $67.40 \pm 6.11$ years, the mean height was $152.90 \pm 5.93 \mathrm{~cm}$, and the mean weight was $56.00 \pm 7.70 \mathrm{~kg}$. The inclusion criteria for the study subjects were no clinical problems performing resistive muscle strengthening exercises using elastic bands, not performing regular exercise, and not having previously undergone any arthritis-related surgical operation. The subjects Kellgren-Lawrence (K/L) grade of the knee joints was 2 or higher and their visual analog scale results for knee pain were 5 points or lower. They were randomly and equally assigned to three groups: CKCEG, OKCEG, and CG. Ethical approval for the study was granted by the Youngdong University institutional review board.

During the exercise program, the CKCEG, OKCEG, and CG performed their exercises three times per week for four weeks. Each exercise session included a warming-up exercise, the main exercise, and a cooling-down exercise. The total exercise time for each session was $20 \mathrm{~min}$. The warming-up exercises and the cooling-down exercises were performed for $5 \mathrm{~min}$ each. The main exercises consisted of a total of three sets, consisting of 10 repetitions of the exercises.

Different colored elastic bands denote the degree of elasticity. The CKCEG and the OKCEG used red elastic bands (Hygenic Corporation, USA), which have medium resistance. The CG performed quadriceps strengthening exercises.

In the CKCEG, the subjects wore a Body Safe Belt (Black
Diamond Equipment Ltd, USA) around the lumbar region that was connected to the elastic bands. The lower ends of the elastic bands were attached to the subjects' shoes or their bare feet before performing the exercises. During the first and second week, knee joint flexion and extension were performed simultaneously with hip joint flexion and extension in minisquat exercises. During the third and fourth weeks, squat exercises were performed by those subjects who were able to do so. In the OKCEG, the subjects adopted a prone position, and one end of the elastic band was attached to the wall, and the other end was attached to their ankles. The subjects then performed knee joint flexion and extension. As resistance varies with the length of the bands, the length of the bands was adjusted to allow the subjects to pull the bands 10 times with the same motion. The subjects in the CG performed the quadriceps strengthening exercises in a supine position with a towel rolled in the shape of a cylinder. The towel was placed below their knees, and the subjects then slowly extended their knees while maintaining ankle dorsiflexion. The exercise time depended upon the age of the subjects and their levels of fatigue.

An MP 35 (Biopac System, USA) was used to measure the electromyographic activityes of the quadriceps femoris muscle. Surface electrodes were attached to the vastus medialis (VM), the rectus femoris (RF), the vastus lateralis (VL), the semitendinosus (ST), and the biceps femoris (BF). Raw EMG signals were sampled at $1,000 \mathrm{~Hz}$ and full-wave rectified. A $30 \mathrm{~Hz}$ low-pass filter was then applied to the root mean square (RMS) signals, which were then sampled between 40 to $250 \mathrm{~Hz}$ and stored in a computer file. A $60 \mathrm{~Hz}$ notch filter was used to remove unnecessary signals because the Korean electricity supply an alternating current frequency of $60 \mathrm{~Hz}$. To stabilize variance in the collected data, the raw measurement data were converted into root mean squares (RMS), and the values of the middle $3 \mathrm{sec}$ of $5 \mathrm{sec}$ a measuring time, excluding the first and last second, were used in the analysis. The RMS values obtained were divided by the maximum voluntary isometric contraction (MVIC) value to normalize the values to \%MVIC values within a range of $0-100 \%$.

In this study, the paired sample t-test was conducted to examine changes in the electromyographic activities of the muscles within the groups during the treatment period. One-way ANOVA was conducted to examine significant differences in muscle activities among the groups. The Bonferroni correction method was used as a post hoc test. SPSS 12.0 for Windows was used for the statistical processing of the data, and a significance level of 0.05 .

\section{RESULTS}

According to the results of the comparisons within the groups, the CKCEG showed significant increases in the electromyographic activities of the VM, RF, VL, ST, and $\mathrm{BF}$; the OKCEG displayed significant increases in the electromyographic activities of the $\mathrm{VM}, \mathrm{RF}, \mathrm{ST}$, and $\mathrm{BF}$; and the $\mathrm{CG}$ exhibited significant increases in the electromyographic activities of the RF, VL, ST, and BF $(p<0.05)$. In the comparisons between the groups after the treatment, the 
electromyographic activities of the VM, RF, and VL were significantly higher in the CKCEG than in the CG, and the electromyographic activities of the VM, RF, and ST were significantly higher in the OKCEG than in the CG $(p<0.05)$ (Table 1).

\section{DISCUSSION}

If patients' activities of daily living are restricted due to pain associated with degenerative gonarthritis, movements will become further restricted due to joint deformation, and the formation of osteophytes on articular surfaces will further restrict activities, forming a vicious circle. Therefore, the maintenance of activity through exercise therapy has been presented as a major treatment option for degenerative gonarthritis. Among various treatment methods, muscle strength training using elastic bands has been frequently used as a muscle strengthening exercise for elderly persons. Such exercise is used not only because of its simplicity and low cost, but also because of its ability to strengthen the musculoskeletal system of the lower extremities. Thus, it is the most suitable resistive exercise program for elderly persons, enabling muscle strengthening with a reduced burden on the joints ${ }^{7}$.

Jo and Jung ${ }^{8)}$ analyzed changes in the electromyographic activities of 17 healthy males who performed OKCEs and CKCEs as part of an isotonic exercise regime. They reported that the electromyographic activity of the long head of the BF muscle increased significantly in the OKCEG. $\mathrm{Kim}^{9)}$ suggested that CKCEs are useful for selective training of the gluteus medius muscle and that OKCEs are desirable for gradual training in the early stage of rehabilitation. $\mathrm{Han}^{10)}$ reported that subjects in an exercise group who participated in CKCEs showed greater increases in the electromyographic activities of their left lower extremities than those in a group who performed OKCEs. They attributed this finding to the body segments being fixed during CKCEs. Kwon et al. ${ }^{11)}$ reported that when 41 normal adults performed OKCEs and CKCEs for six weeks, the OKCEs were more effective at strengthening the VM muscle and the anterior tibial muscle during the first two weeks of exercises. They also found that the CKCEs were more effective in strengthening the vastus lateralis muscle, the hamstring muscle, and the gastrocnemius muscle after two weeks.

In the study of $\mathrm{Kim}^{12)}$, elderly persons performed knee joint muscle strengthening exercises using elastic bands, and their lower extremity muscle strength increased compared to before the exercise after performing the exercises for five weeks. Yun ${ }^{13)}$ reported that chronic gonarthritis patients who performed isometric exercises and isotonic exercises using elastic bands for five weeks showed significant increases in the electromyographic activities of the quadriceps femoris muscle and the hamstring muscle following both isometric exercises and the isotonic exercises using elastic bands. In comparisons between the groups, the isotonic exercises using the elastic bands were more effective than the isometric exercises at strengthening the quadriceps femoris muscle and the hamstring muscle of the chronic gonarthritis patients. Mikesky et al. ${ }^{14)}$ divided elderly per-
Table 1. Changes in the electromyographic activities of the $\mathrm{VM}, \mathrm{RF}$ and $\mathrm{VL}, \mathrm{ST}$, and BF between the groups (Unit:\%)

\begin{tabular}{|c|c|c|c|}
\hline Muscle & Group & Pre & Post \\
\hline \multirow[t]{3}{*}{$\mathrm{VM}^{\dagger}$} & CKCEG** $^{* *}$ & ${ }^{\mathrm{a}} 19.0 \pm 8.5$ & $37.0 \pm 21.6$ \\
\hline & OKCEG** & $15.9 \pm 4.4$ & $34.3 \pm 16.5$ \\
\hline & $\mathrm{CG}$ & $16.6 \pm 6.1$ & $18.8 \pm 6.1$ \\
\hline \multirow[t]{3}{*}{$\mathrm{RF}^{\dagger}$} & $\mathrm{CKCEG}^{* *}$ & $20.5 \pm 8.5$ & $34.6 \pm 14.1$ \\
\hline & OKCEG* & $19.7 \pm 8.9$ & $31.1 \pm 14.8$ \\
\hline & $\mathrm{CG}^{*}$ & $17.2 \pm 6.1$ & $20.5 \pm 5.1$ \\
\hline \multirow[t]{3}{*}{$\mathrm{VL}^{\dagger}$} & $\mathrm{CKCEG}^{* *}$ & $8.6 \pm 5.9$ & $22.8 \pm 7.9$ \\
\hline & OKCEG & $10.2 \pm 4.1$ & $13.7 \pm 8.6$ \\
\hline & $\mathrm{CG}^{* *}$ & $7.9 \pm 3.5$ & $12.7 \pm 4.6$ \\
\hline \multirow[t]{3}{*}{$\mathrm{ST}^{\dagger}$} & $\mathrm{CKCEG}^{* *}$ & $26.5 \pm 20.0$ & $36.9 \pm 27.6$ \\
\hline & OKCEG** & $28.5 \pm 19.7$ & $67.9 \pm 35.2$ \\
\hline & $\mathrm{CG}^{* *}$ & $13.4 \pm 8.7$ & $22.1 \pm 17.4$ \\
\hline \multirow[t]{3}{*}{$\mathrm{BF}$} & $\mathrm{CKCEG}^{* *}$ & $19.8 \pm 10.0$ & $28.4 \pm 14.2$ \\
\hline & OKCEG** & $24.0 \pm 14.1$ & $39.2 \pm 18.8$ \\
\hline & $\mathrm{CG}^{*}$ & $12.6 \pm 7.1$ & $22.4 \pm 14.1$ \\
\hline
\end{tabular}

VM: vastus medialis, RF: rectus femoris, VL: vastus lateralis, ST: semitendinosus, BF: biceps femoris, CKCEG: close kinetic chain exercise group, OKCEG: open kinetic chain exercise group, CG: control group, ${ }^{\mathrm{a}}: \mathrm{Mean} \pm \mathrm{SD},{ }^{*}$ : Paired t- test, ${ }^{\dagger}$ : One way ANOVA, ${ }^{*}$ : $\mathrm{p}<0.05,{ }^{* *}: \mathrm{p}<0.01$

sons aged 65 years or more into an exercise group which performed gradual muscle strengthening exercises using elastic bands and a non-exercise group. They reported that the exercise group showed an increase of $12 \%$ in the muscle strength of the knee joint extensor muscles and an increase of $10 \%$ in that of the knee joint flexor muscles. Damush and Damush $^{15)}$ investigated the effects of resistance exercises using elastic bands on the muscle strength and health-related quality of life of elderly women aged 68 years on average. Their subjects showed increases in the muscle strength of the latissimus dorsi muscle, the pectoralis major muscle, and the quadriceps femoris muscle of $15 \%, 13 \%$, and $25 \%$, respectively, but there were no significant changes in their mental and physical health functions. Topp et al. ${ }^{16)}$ reported that when elderly persons aged 65 years or more performed elastic band exercises, their gait and balance abilities improved. Krebs et al. ${ }^{17)}$ reported that 132 elderly persons with functional restriction, who performed muscle strengthening exercises using elastic bands, showed increases in the strength of the lower extremity muscles (hip joint abductor muscles, extensor muscles, and knee joint extensor muscles) of $17.6 \%$ and $7.3 \%$ in the exercise group and the control group, respectively, after the exercises compared to before the exercises. Deyle et al. ${ }^{18)}$ reported that when chronic gonarthritis patients performed muscle strengthening exercise using elastic bands for four weeks, their gait and functions improved. According to the results of our present study, in the comparisons within the groups, the CKCEG showed significant increases in the electromyographic activities of the VM, the RF, the VL, the ST, and BF; the OKCEG showed significant increases in the electromyographic activities of the VM, the RF, the ST, and the BF; and the CG 
showed significant increases in the electromyographic activities of the RF, the VL, the ST, and the BF. In comparisons between the groups after the treatment, the CKCEG showed significantly higher electromyographic activities of the VM, the RF, and the VL than the CG, and the OKCEG showed significantly higher electromyographic activities of the VM, the RF, and the ST than the CG. Studies by Han ${ }^{19)}$ and Nougarou ${ }^{20}$ ) revealed that an increase in muscle activity was associated with an increase in muscle strength. In the present study, muscle activity was a greater after the exercise than prior to the exercise.

The results of the present study are in agreement with other published results in the literature and suggest that CKCEs or OKCEs using elastic bands are considerably more effective than quadriceps strengthening exercises at strengthening lower extremity muscles. They also indicate that when patients are able to perform selective muscle strengthening exercises, exercises tailored to such patients should be recommended. As single joint exercises, OKCEs are considered very useful for patients with limited ranges of joint motion. They are also useful in early stage muscle strengthening programs, in individual muscle training, and in multi-joint exercises. CKCEs are suitable for stabilization and functional training.

This study had some limitations. First, as the number of subjects with degenerative gonarthritis was limited, the interpretation of the results presented herein cannot be generalized. Second, the right lower extremity was the dominant side all the subjects, and only the muscle strength on that side was measured. Thus, the potential effect of the exercises on changes in the muscle strength on the other side is not known. In later studies, appropriate muscle strengthening exercises using elastic bands should be performed not only by chronic degenerative gonarthritis patients, but also by acute gonarthritis patients, and the effects of the exercises should be examined.

\section{REFERENCES}

1) Jones CJ, Rose DJ: International guidelines for training physical activity instructors for older adults. J Aging Phys Act, 2004, 12: 1-2. [Medline]

2) Kaufman KR, Hughes C, Morrey BF, et al.: Gait characteristics of patients with knee osteoarthritis. J Biomech, 2001, 34: 907-915. [Medline] [CrossRef]

3) Hurley MV: The role of muscle weakness in the pathogenesis of osteoarthritis. Rheum Dis Clin North Am, 1999, 25: 283-298, vi. [Medline]
[CrossRef]

4) McAlindon TE, Cooper C, Kirwan JR, et al.: Determinants of disability in osteoarthritis of the knee. Ann Rheum Dis, 1993, 52: 258-262. [Medline] [CrossRef]

5) McCarthy LH, Bigal ME, Katz M, et al.: Chronic pain and obesity in elderly people: results from the Einstein aging study. J Am Geriatr Soc, 2009, 57: 115-119. [Medline] [CrossRef]

6) Thomas VS, Hageman PA: Can neuromuscular strength and function in people with dementia be rehabilitated using resistance-exercise training? Results from a preliminary intervention study. J Gerontol A Biol Sci Med Sci, 2003, 58: 746-751. [Medline] [CrossRef]

7) Kim D, Kim J: The effects of weight training and elastic band for the elderly. The Korea J Sports Sci, 2010, 19: 1103-1115.

8) Jo J, Jung S: Electromyographic analysis of isotonic closed and open kinetic chain exercises. The Korean Journal of Physical Education, 2001, 40: 633-643.

9) Kim E: The effect of closed and open kinetic chain exercises on the activation of hip abductors. Inje Unversity, Dissertation of master's degree, 2006.

10) Han H: Effect of close kinetic chain and open kinetic chain position on proprioceptive neuromuscular facilitation applied to the unilateral upper extremity on the muscle activation of lower extremity. Korea University, Dissertation of master's degree, 2009.

11) Kwon Y, Park S, Kim K: The effect of open and closed chain exercise on lower extremity muscle activity in adults. Journal of the Korean Society of Physical Medicine, 2012, 7: 173-182.

12) Kim H: The effect of knee muscle power strengthening using Thera Band on the balance control ability in the elderly. Dankook University Dissertation of master's degree, 2003.

13) Yun H: The effects of isometric exercise and isotonic exercise using elastic band on strength, range of motion, standing balance for the patient with chronic knee arthritis. Dankook University, Dissertation of master's degree, 2006.

14) Mikesky AE, Topp R, Wigglesworth JK, et al.: Efficacy of a home-based training program for older adults using elastic tubing. Eur J Appl Physiol Occup Physiol, 1994, 69: 316-320. [Medline] [CrossRef]

15) Damush TM, Damush JG Jr: The effects of strength training on strength and health-related quality of life in older adult women. Gerontologist, 1999, 39: 705-710. [Medline] [CrossRef]

16) Topp R, Mikesky A, Wigglesworth J, et al.: The effect of a 12-week dynamic resistance strength training program on gait velocity and balance of older adults. Gerontologist, 1993, 33: 501-506. [Medline] [CrossRef]

17) Krebs DE, Jette AM, Assmann SF: Moderate exercise improves gait stability in disabled elders. Arch Phys Med Rehabil, 1998, 79: 1489-1495. [Medline] [CrossRef]

18) Deyle GD, Henderson NE, Matekel RL, et al.: Effectiveness of manual physical therapy and exercise in osteoarthritis of the knee. A randomized, controlled trial. Ann Intern Med, 2000, 132: 173-181. [Medline] [CrossRef]

19) Han JS.: Investigation of the relationship between muscle force and electromyography during dynamic mouvement of elbow joint. J Biomed Eng Res, 22: 211-216.

20) Nougarou F, Dugas C, Deslauriers $C$, et al.: Physiological responses to spinal manipulation therapy: investigation of the relationship between electromyographic responses and peak force. J Manipulative Physiol Ther, 2013, 36: 557-563. [Medline] [CrossRef] 